\title{
REMOVAL OF CLOUD AND SHADOW INFLUENCE FROM REMOTELY SENSED IMAGES THROUGH LANDSAT8/OLI/TIRS USING MINIMUM DISTANCE SUPERVISED CLASSIFICATION
}

\author{
Renuka S. Gound \\ Research Scholar, Computer Engineering, PCCOE, Nigadi, \\ Pune, Maharashtra 411044, India \\ renuka060182@gmail.com \\ Dr. Sudeep D. Thepade \\ Professor, Computer Engineering, PCCOE, Nigadi \\ Pune, Maharashtra 411044, India \\ sudeep.thepade@pccoepune.org
}

\begin{abstract}
Earth Coverage Antennas (ECA) belonging to LANDSAT8/OLI/TIRS is utilized for coverage of ground sites or land covers below Spacecraft. Earth observation system is a primary application of ECA. Earth Observation is needed for monitoring and adapting the global changes which may occurs through environmental effects over land cover. Natural or human-induced disasters, weather, ocean, climate, land is need to be understood for adaption of global change. Classification of land cover images captured through Earth Coverage Antennas provide necessary information required for decision making. Application of land cover classification includes making report for land cover change, vegetation, prediction of environmental or climate issue, meteorology, etc. Present article explores application of supervised classification over land cover images captured through Landsat 8/OLI/TIRS with improvement in the visibility of Land Cover Surface by removing the obstacles like cloud and their shadows which are coming in between satellite and land surface. Supervised classification used in experimentation is Minimum Distance by using semi-automatic classification plugin (SCP) with QGIS. Accuracy assessment is done for evaluation of error and reliability of land cover classification. Accuracy Assessment of supervised classification applied over land cover includes calculation of Kappa Hat Statistics, UA (User Accuracy), PA (Producer Accuracy), Error of Omission and Error of Commission. Overall Accuracy seen in the derived Classification result and statistics is more desirable.
\end{abstract}

Keywords: Earth Coverage Antennas (ECA); Minimum Distance Classification; Landsat 8/OLI/TIRS; Quality Assessment Band; UA (User Accuracy); PA (Producer Accuracy); Kappa Hat Statistics

\section{Introduction}

Antenna Systems are very important for earth observatory system, remote sensing, detecting and removing images to transmit and receive the signals and data.[24] LANDSAT8/OLI/TIRS is a Remotely Sensed Earth Observatory System. It was launched on February 11, 2013. It carries the Operational Land Imager and the Thermal Infrared Sensor.

OLI is an Optical Land Imaging Sensor which is utilized for cirrus cloud detection and investigation over water Resources. Band 1 and Band 9 are respectively used for this specific purpose. TIRS is Thermal Infrared Sensor whose functionality is acquiring data of resolution of 100 meter. OLI and TIRS are carried by spacecraft. GNE is Ground Network Element who receives data at ground station antenna from Spacecraft. MOE is Mission Operations Element which is having a capability of collecting and scheduling earth observatory scenes. DPAS is Data Processing and Archive System which is responsible for archiving data, data calibration, data processing and data distribution.

As it can be observed from Fig.1b. how data is collected with spacecraft and antenna systems. 


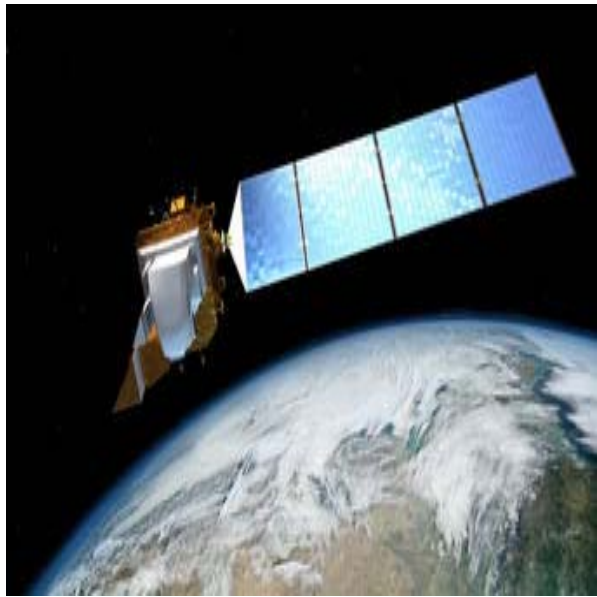

(a) Satellite in Orbit

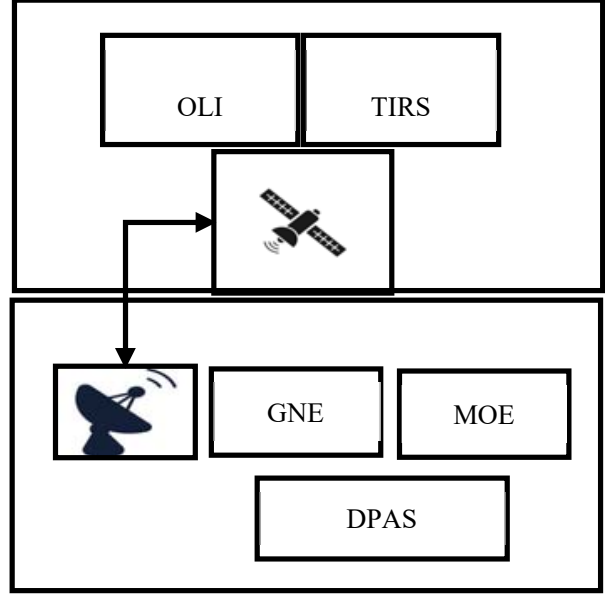

(b) Components of Earth Coverage Antenna

Fig. 1. Illustration of LANDSAT 8 /OLI/TIRS [1]

Proposed system captures scenes of study area which are remotely sensed through LANDSAT8 /OLI/TIRS in different wavelengths. Images are acquired on different dates, so as to suppress the influence of clouds and shadows on ground surface.

Proposed method of cloud and shadow removal mainly focusses on pre-processing of Band-2 to Band-7 images of targeted land cover area using Atmospheric Correction, Classification of land cover with NDVI enhancement [6], Identification of value of cloud and shadow pixels with BQA, Reconstruction of a target image through reference images of same area. Supervised classification in remote sensing [3], supervises each pixel in an image. Known land cover type which contains values of pixel or spectral signatures related with every class is used as training sample and then whole image is classified using this pixel values or spectral signatures. Overlapping of classes should be avoided or it should be minimal.

Images of same area is captured for classification of land cover surface. Classification is mainly focusing on identification of different components available on Land Surface. Cloud and shadow pixels are identified from target image. These cloud and shadow contaminated pixels are replaced with reconstruction of an image through reference images of same area.

Accuracy assessment can be used here to evaluate pixels which are correctly classified with an error occurred by misclassification of a pixel. Experimentation is performed by using Semi Classification plugin in QGIS [29].

Fig. 2 (a) Illustrates effect of clouds and their shadows on Landcover surface captured with Landsat 8/OLI/TIRS [1], 2 (b) shows Classification of land cover surface 2 (c) shows Cloud and Shadow Mask prepared to remove cloud and shadow contaminated pixels from land cover surface and 2 (d) shows resultant image after removing cloud and shadow contamination pixels. As visibility of land cover surface is improved then applicability of image can be increased for various application such as observation of change of land cover detection.

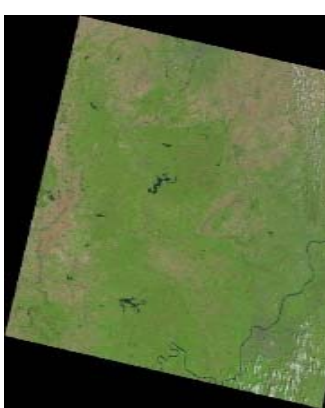

(a)

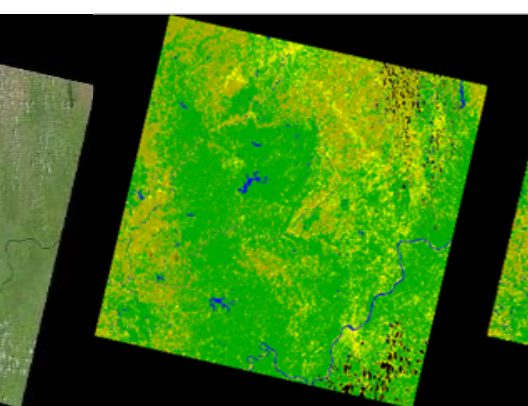

(b)

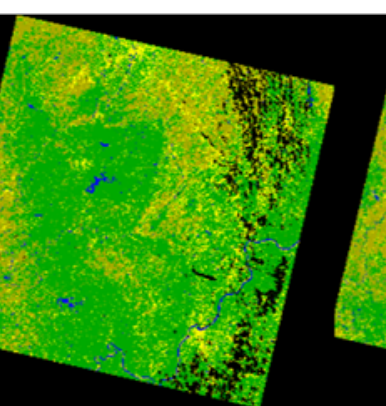

(c)

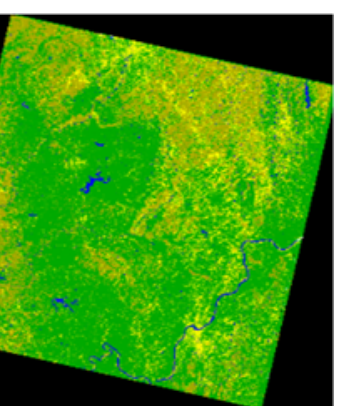

(d)

Fig. 2. Removing Clouds and Shadows from classification image of Land Cover Surfaces acquired by Landsat 8/OLI/TIRS a) Input Image with Clouds and Shadows [1] b) Classification of an input image c) Cloud and Shadow Mask of an Input Image d) Removing Clouds and Shadows from Classification Image.

Key contribution of paper is given below

- Acquiring Images of study area with different cloud coverages from LANDSAT8/OLI/TIRS in different wavelengths specifically in Band-2 to Band-7 and Quality Assessment Band (BQA).

- Conversion of DN to actual surface reflectance known as Atmospheric Correction of Band-2 to Band-7 images of targeted Land cover 
- Application of Minimum Distance Algorithm to Classify targeted image into five classes.

- Enhancing Classification targeted image with NDVI calculation

- Removal of cloud and shadow pixels by using BQA of targeted image

- Reconstruction of a Target Image by using Reference Image.

- Accuracy Assessment of Classification result.

Present article is organized as follows. Architecture of Proposed system of an application of Supervised classification of Land Cover to generate Cloud and Shadow Mask to Remove Cloud and Shadow contaminated pixels with Reconstruction of Cloud and Shadow free Image is elaborated in Section 2. Downloading study area of land cover with different bands needed for identification of cloud and shadow contaminated pixels is shown in Section 3, Pre-processing of Landsat8 OLI/TIRS image is exhibited through 4, Essential tasks require to perform Supervised Classification are explained in Section 5. Section 6 Demonstrates Procedure of cloud and shadow masking. Removal and replacement of cloud and shadow contaminated pixels procedure is exhibited across section 7. Accuracy assessment report of classification result is shown in section 8 .

\section{Architecture of Proposed Method}

Proposed method of "Classification of Land Cover Surface to detect and remove influence of cloud and shadow for improving the visibility of land cover" is elaborated in Fig. 3.

Fig. 3 exemplifies steps involved from downloading study area to generating mask needed for removal of cloud and shadow pixels.

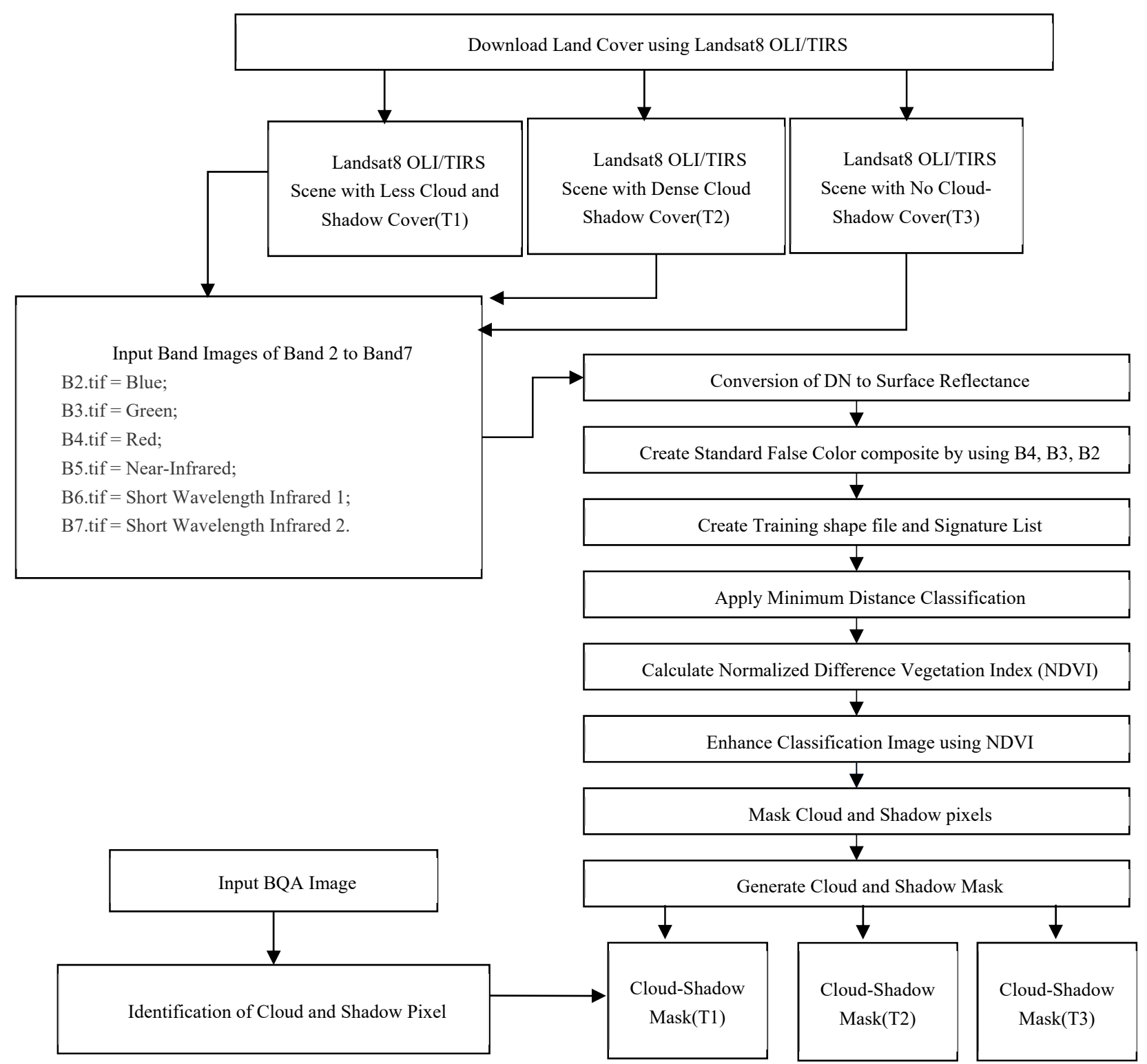

Fig. 3. Architecture of "Classification of Land Cover Surface to detect and remove influence of cloud and shadow for improving the visibility of land cover" 
Band wise images are needed to be downloaded from https://earthexplorer.usgs.gov/. For Experimentation Images of study area are acquired on different dates with less, dense and no Cloud and Shadow Coverage in different wavelengths (Band-2 to Band-7 and BQA). Images are affected by atmospheric noise interference so; Preprocessing is required for Atmospheric Correction. After preprocessing Band-2 to Band-7 images are selected for further processing. Natural color composite and false color composites are required for classification process. ROI (Region of Interest) are selected from natural color composite and false color composite, are saved into training shape file. To observe spectral differences of ROIs for classification process; signature list is needed. Signature list collects spectral characteristics of different components identified for classification. Supervised Classification using Minimum Distance algorithm [8] is applied to classify land cover surface. Classification is enhanced with NDVI [6] calculation. BQA is used to identify pixel values related to clouds, shadows, clear area. These pixel values are selected from classification image so as to prepare Cloud and Shadow mask for each downloaded image from LANDSAT8 OLI/TIRS.

By using Cloud and Shadow Masks generated for all the scenes acquired on different dates are used for removal of clouds and shadows from classification Image.

\section{Study Area}

Study Area used in this experimentation is places around Bloomington. This city is known as "City of Trees". Most of the city is covered with forests, rivers, with less construction, for classification purpose it will be useful to make different categories such as water, vegetation, soil and human made constructions etc. 'Bloomington is located in the county seat of Monroe County in the central region of the U.S. state of Indiana'. Dataset is downloaded from https://earthexplorer.usgs.gov/.

Details of Land Cover are as follows; Landsat Scene ID is "LC80210332017116LGN00", Output Format is "GEOTIFF", Spacecraft ID is "LANDSAT_8", Sensor ID is "OLI_TIRS", WRS_PATH is 21 and WRS_ROW is 33.[28]

Land cover shown in Fig. 4 (a) is acquired on 31-7-2017 with 10.15\% cloud coverage. This one is a first targeted image, from which cloud and shadow pixel will be identified in the experimentation and then that contaminated area by cloud and shadow pixels will be replaced with the pixels from land covers acquired on 26-4-2017(Second Targeted Image) shown in 4 (b) and 29-4-2018(Reference Image) with 0.03\% Cloud Cover shown in 4 (c). Same process will be applied on Second Target Image to remove cloud and shadow and reconstruction. Downloaded Images of Land Cover Surface needs to be pre-processed. Pre-processing for landsat8 bands of landcover scenes is described in next session.

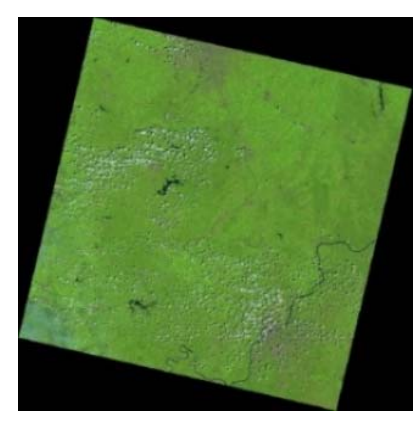

(a)

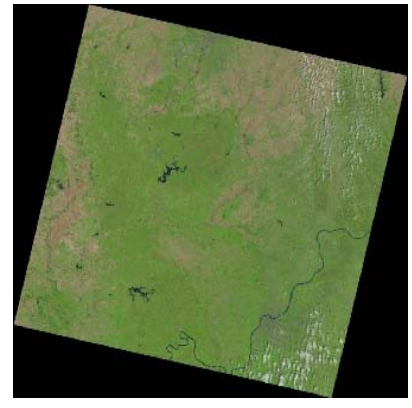

(b)

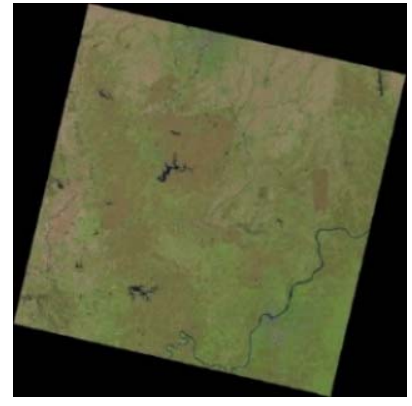

(c)

Fig. 4. Landsat Scene "LC80210332017116LGN00" through LANDSAT8/OLI/TIRS (a) Acquired on 31-7-2017 with 10.15\% Cloud Cover (b) Acquired on 26-04-2017 with 3.42\% Cloud Cover (c) Acquired on 29-4-2018 with $0.03 \%$ Cloud Cover.

\section{Pre-processing of Landsat8 OLI/TIRS image}

In remote sensing process, reflectance values captured through satellite or sensors are getting affected through interference caused by atmosphere. These values may create problems in further processing of these images therefore removal of atmosphere interference with reflectance values is necessary. This process of removing atmospheric interference to get actual reflectance of surface components is known as Conversion of DN to Surface Reflectance

Satellite generated Digital Numbers (DN) represents Multispectral Image Information. 
As it may be observed from Fig. 5. Energy from the outside view can affect thermal data received by sensor. Radiance is the energy received at satellite which is affected by outside energy, it depends on reflectance. Atmospheric Correction is a process to convert DNs to actual ground or surface reflectance.

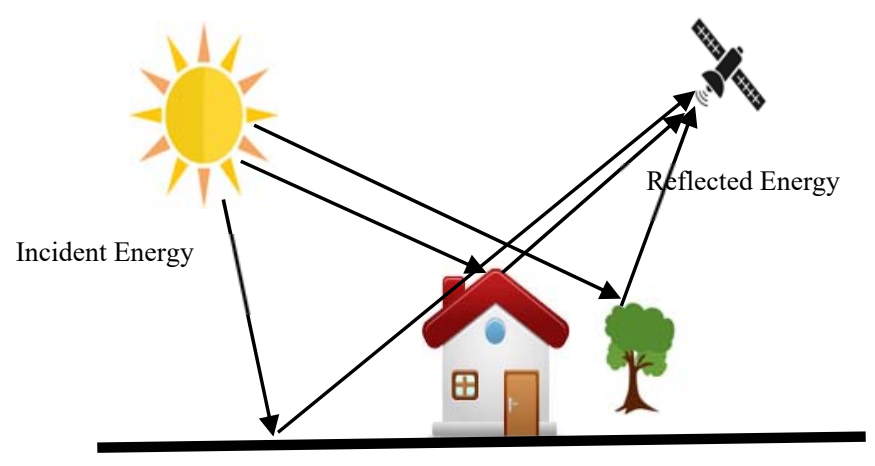

Fig. 5. Conversion of DN to Surface Reflectance

Proposed system makes use of Dark Object Subtraction (DOS1) [4] to convert DN to surface reflectance of components available on Land Surface.

Fig. 6. (a-f) shows respectively Band 2 to Band 7 images received at sensor, with atmospheric interference. These captured images are darker due to effect of interference of atmospheric noise, so it is necessary to reduce atmospheric scattering effect. To remove atmospheric scattering, it is required to subtract dark pixels from band images.

DOS1 is an image-based technique which works on strategy as of due to environmental effects, Dark Objects from captured images of satellite will provide more information of atmospheric scattering. DOS1 searches for dark pixel values and subtracts these values from each band to remove atmospheric scattering.

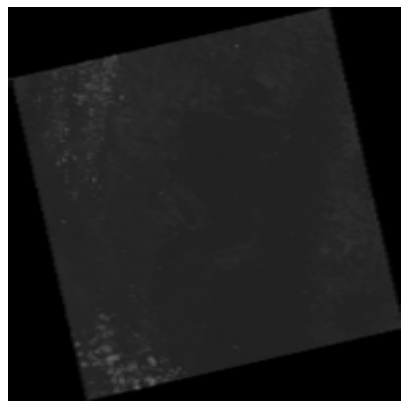

(a)

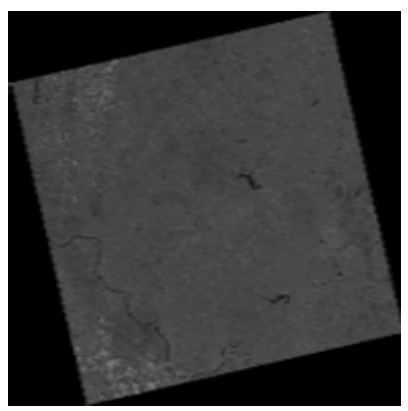

(d)

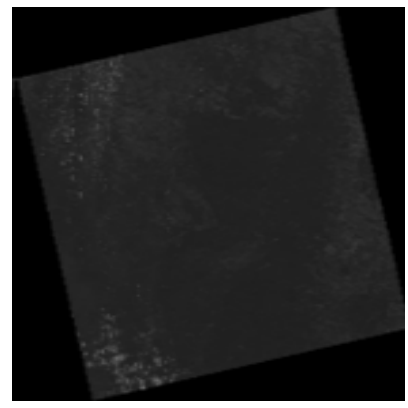

(b)

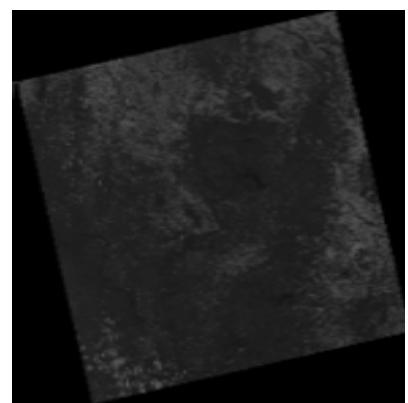

(e)

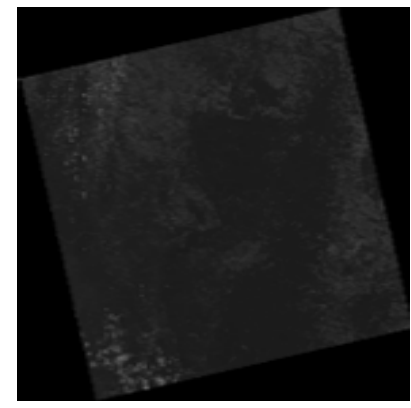

(c)

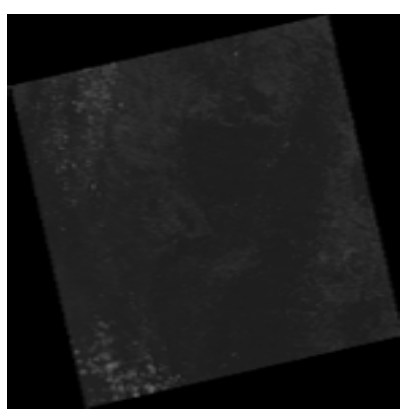

(f)

Fig. 6. Band Images before DOS1 (a) Band2, (b) Band3, (c) Band4, (d)Band5, (e) Band6, (f) Band7

Fig. 7 (a-f) shows Reflectance images of Band-2 to Band-7 respectively; with actual ground reflectance without any outside energy interfere, after application of DOS1.

Images obtained after execution of DOS1 technique are free of atmospheric scattering and can be utilized for further processing. Next section describes, way of applying supervised classification on reflectance images obtained. 


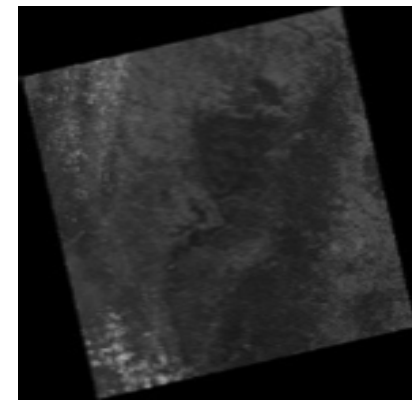

(a)

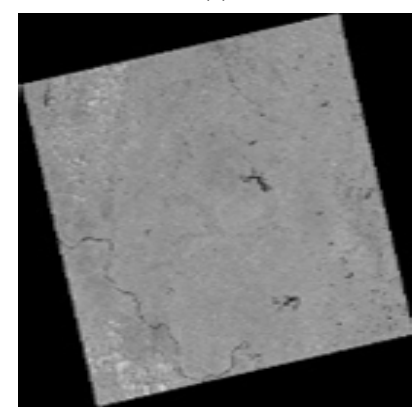

(d)

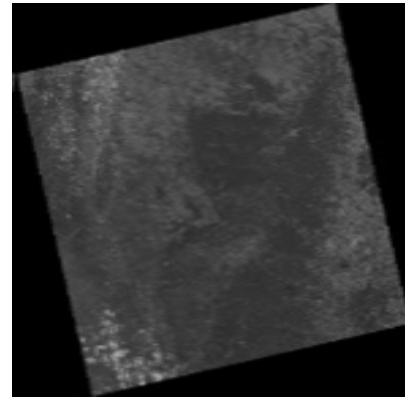

(b)

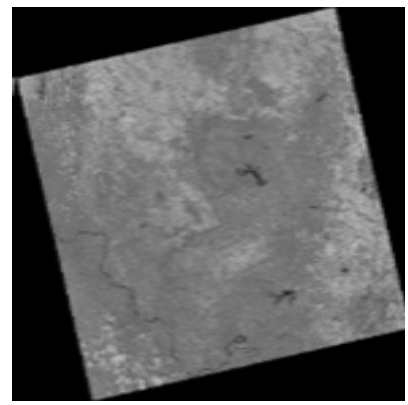

(e)

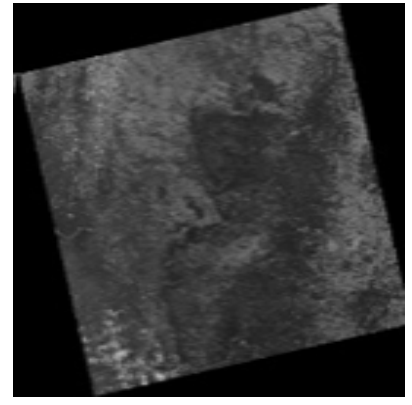

(c)

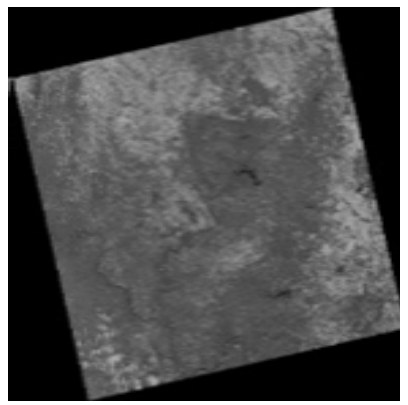

(f)

Fig. 7. Reflectance Images after DOS1 (a) Band2, (b) Band3, (c) Band4, (d)Band5, (e) Band6, (f) Band7

\section{Supervised Classification of Land Cover}

Supervised classification [23] in remote sensing images to find material on ground surface as per their spectral signatures. After an application of supervised classification over remotely sensed image, it produces thematic map of ground surface.

Requirements of supervised classification of land cover are given as following

\subsection{Land Cover}

"Land cover is the observed (bio)physical cover on the earth's surface". Land cover is generally described by using Vegetation and human- made entities like constructions. Land is by its own is used to describe its surface which includes bare soil, Water Surface, bare rock.

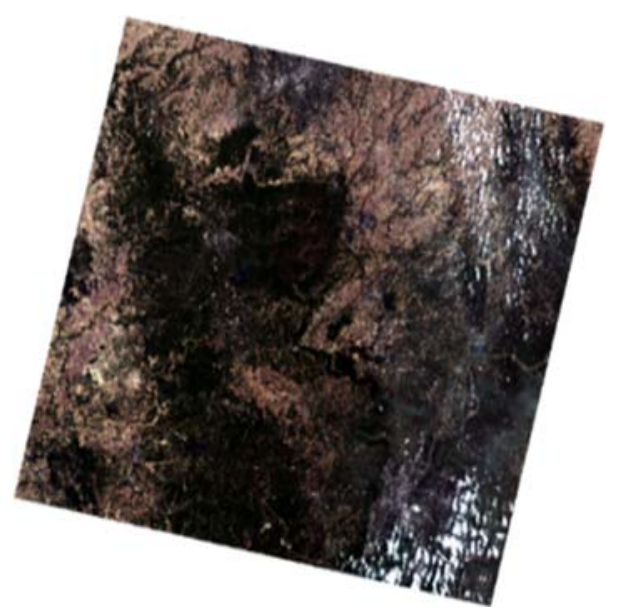

(a)

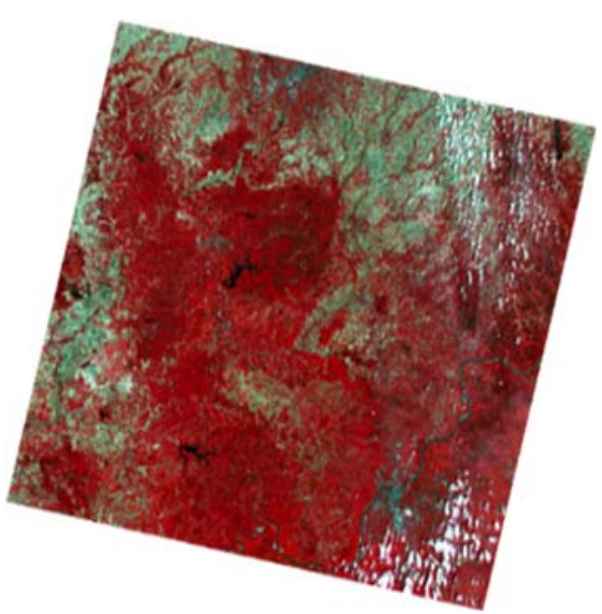

(b)

Fig. 8. Images of Study Area (a) Natural Color Composite (B4,B3,B2) (b) False Color Composite (B5,B4,B3).

In general, for classification of Land cover, the entities of land surface such as water, soil, vegetation and human made entities like built-up or various constructions are taken into considerations. As per the resolution of sensor classes can vary. 
Fig. 8 shows images of land-cover of study area by using Band-4, Band-3, Band-2 create Color Composite of Red, Green and Blue (Fig. 8 (a). Natural Color Composite) and by using Band-5, Band-4, Band-3 create Color Composite of Near-Infrared, Red, and Green. (Fig. 8 (b). False Color Composite). With these Color Composites one can Identify material over ground surface of landcover. Natural Color Composite is also called as true color composite. In False color composite, healthy vegetation is as shown in bright red color, so it can be used to create ROI (Region of Interest) for vegetation class.

\subsection{Create Training shape file}

To create classes of materials in land surface, it is essential to select different regions, this task can be completed by drawing ROIs in the form of polygons over different regions on natural color composite as well as false color composite. Selected ROIs are stored in training shape file. Band wise Spectral Signatures are calculated and stored in Signature List file.

Following ROIs created for classification of Land Cover as shown in Table 1. For experimentation purpose Four classes are identified are Built-up which may include human made entities like various constructions, buildings, road etc., Vegetation regions are identified using Near Infrared Band which consists of grass, trees, gardens etc,

\begin{tabular}{|l|l|l|}
\hline \multicolumn{1}{|c|}{ Class Name } & \multicolumn{1}{|c|}{ Color } \\
\hline Unclassified & 0 & \\
\hline Built-up & 1 & \\
\hline Vegetation & 2 & \\
\hline Soil & 3 & \\
\hline Water & 4 & \\
\hline \multicolumn{2}{|c|}{ Table 1. ROI for Classification }
\end{tabular}

Soil is a region where bare soil or bare rocks are seen over ground surface, Water region contains entities like waterbodies, small ponds, lakes, rivers etc. Regions which were left from classification are clouds and their shadows. These regions are classified as Unclassified region over Land Cover as they are not part of land itself.

\subsection{Minimum-Distance Classification}

Minimum Distance Classification [8][9] is used to assign a pixel to its nearest class. Mean vectors of each class is used for calculation of Euclidean distance of each unknown pixel with it.

Following Equation specifies d (x,y) which is Euclidean Distance between pixel's spectral signature and spectral signature training area.[27]
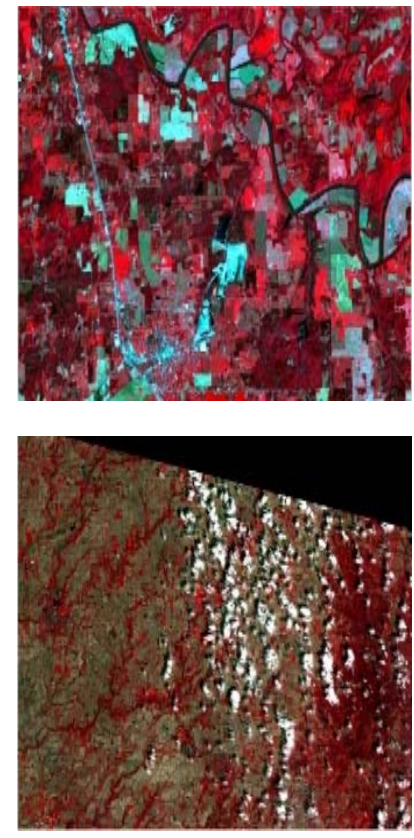

(a)
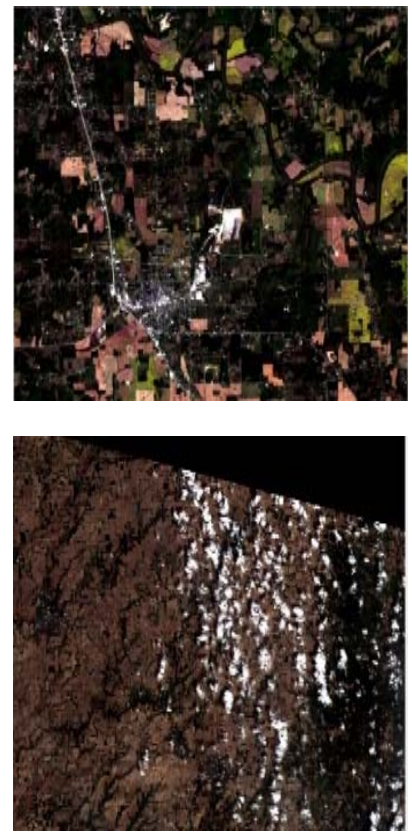

(b)
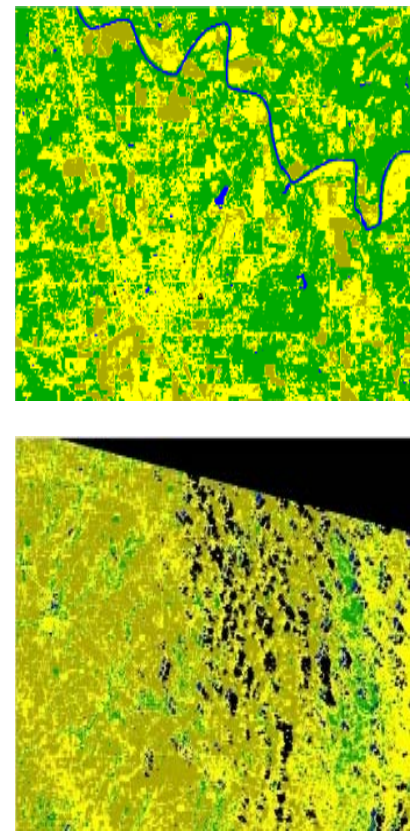

(c)

Fig. 9. Classification Result with different Regions (a) false color composite (b) True color composite and (c) classification result. 
where:

$$
\mathrm{d}(\mathrm{x}, \mathrm{y})=\sqrt{\sum_{\mathrm{i}=1}^{\mathrm{n}}(\mathrm{xi}-\mathrm{yi})^{2}}
$$

- $x i=$ Image Pixel's spectral signature vector;

- $y i=$ Training Area's spectral signature vector;

- $n=$ Total Bands of acquired image.

So, pixel is assigned to class with the basis on spectral signature which one is closer to it by using Euclidean Distance measure.

Fig. 9 Shows, after application of minimum distance classification algorithm, various regions of land cover are classified as per their spectral signatures according to their Euclidean Distance with nearest class. Fig. 9 (a) displays false color composite, Fig. 9 (b) is True color composite and Fig.9 (c) is classification result.

Here, one can observe that as per the classes different regions are classified. Vegetation area which is in bright red color in Fig. 9 (a) is classified in Vegetation class (Green color) in Fig. 9 (c). Construction regions are classified as Built-up and shown in Yellow Color in Fig. 9 (c). Water regions from Fig. 9 (a) and Fig. 9 (b) are classified as Water class in Blue Color in Fig. 9 (c). and Soil is classified as Soil class in Brown Color in Fig.9 (c). Clouds and Shadows are classified as unclassified pixels and represented in black color in Fig. 9 (c).

Result of Minimum Distance Classification is displayed in following Fig. 10.

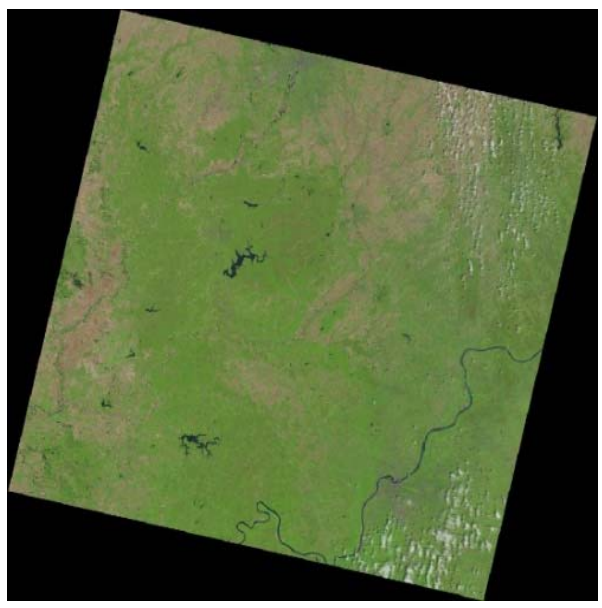

(a)

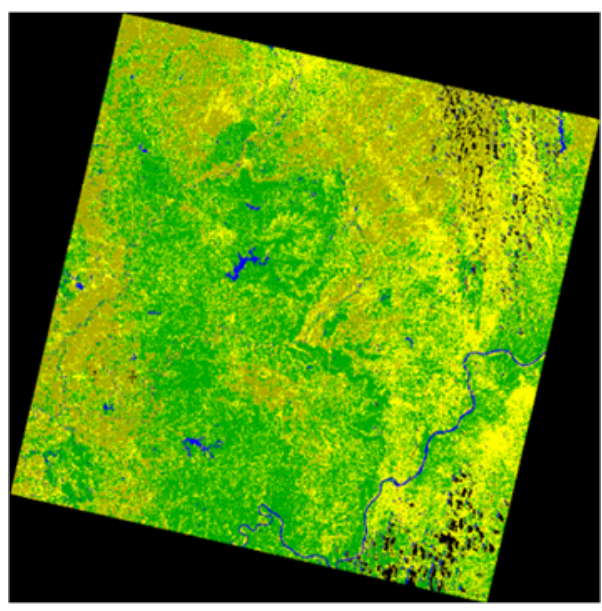

(b)

Fig. 10. Classification Result (a) Input Image Acquired on 26-04-2017 (b) Result of Classification of Input Image

\subsection{Classification Enhancement using NDVI (Normalized Difference Vegetation Index)}

Here NDVI of respective image is used to segment various vegetation areas. With this separation will take place among different regions and classification result will be enhanced.

NDVI [7][10]value is calculated by using Near Infrared Band and Red Band by using following equation (2).

$$
\text { NDVI }=\frac{\text { Near Infrared Band-Red Band }}{\text { Near Infrared Band+Red Band }}
$$

Following are threshold values for NDVI according to type of Vegetation.

\begin{tabular}{|l|l|l|}
\hline \multicolumn{1}{|c|}{ Sr. No. } & \multicolumn{1}{|c|}{ Type of Vegetation } & \multicolumn{1}{c|}{ NDVI Threshold } \\
\hline 1 & Dense & NDVI $>=0.6$ \\
\hline 2 & Moderate & $0.4>$ NDVI $<0.6$ \\
\hline 3 & Sparse & $0.2>$ NDVI $<0.4$ \\
\hline 4 & No vegetation & NDVI $<0.2$ \\
\hline
\end{tabular}

The NDVI value ranges from -1 to 1 . When there is healthy vegetation, NDVI values are high as large amount of Near-Infrared light is reflected from these regions. For unhealthy vegetation it absorbs large amount of NearInfrared light so NDVI value is also low or negative. Resultant Image after application of NDVI calculation on input Image Fig. 11 (a) is shown in Fig. 11 (b).

NDVI value calculated for input image shown in Fig. 11 (a) are as follows -1.0, -0.5, -0.02, 0.4, 0.9 so for enhancing classification threshold value chosen is 0.9 .

After application of threshold value Classification Image Shown in Fig. 12 (a) is enhanced. Result of enhancement of classification is shown in following Fig. 12 (b). 


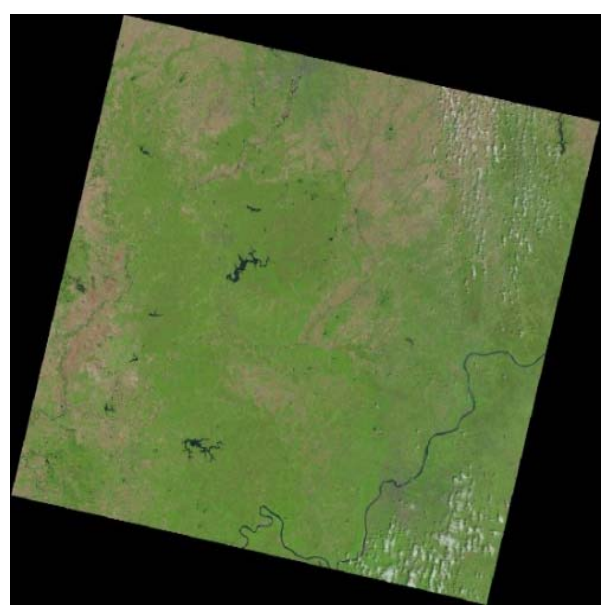

(a)

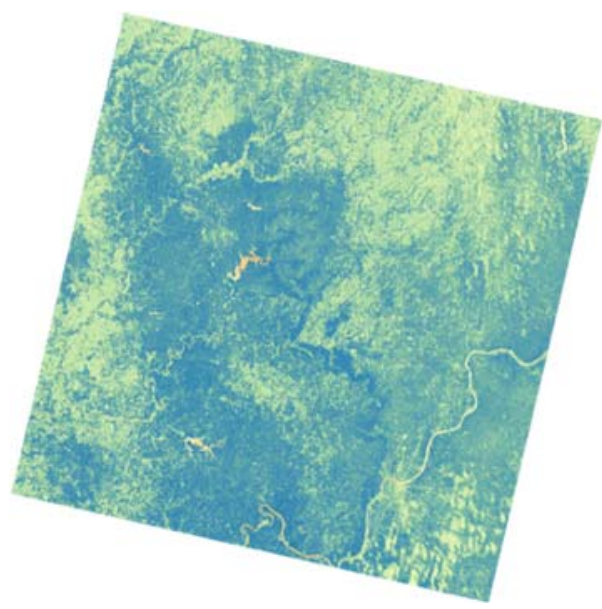

(b)

Fig. 11. NDVI Calculation (a) Input Image Acquired on 26-04-2017 (b) Result of NDVI calculation of Input Image

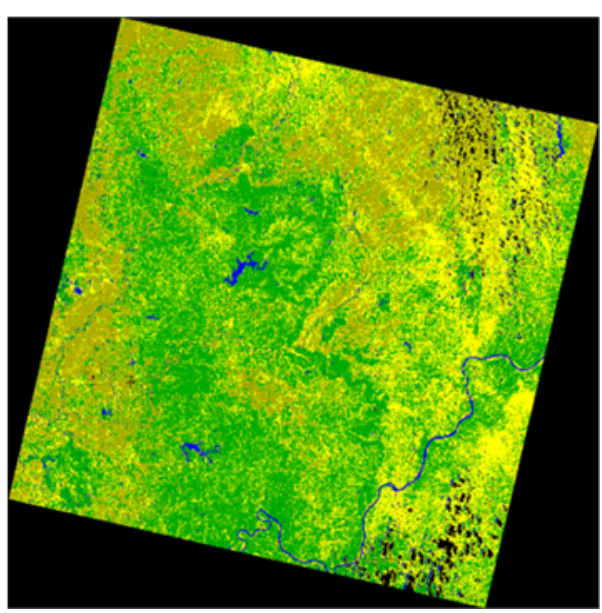

(a)

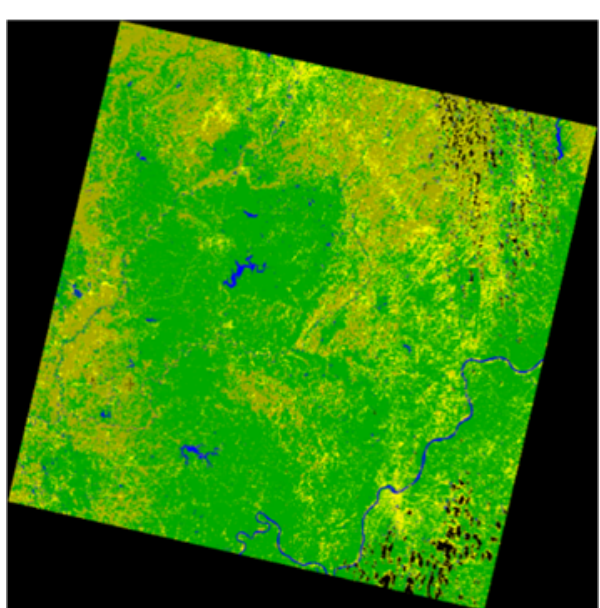

(b)

Fig. 12. Enhancement of Classification (a) Input Image Acquired on 26-04-2017 (b) Result of Enhancement of Classification using NDVI

\section{Procedure to create Cloud-Shadow Mask}

Proposed System makes use of Quality Assessment (QA) bands to detect pixel values of clouds and shadows to create masks of Cloud and Shadow.

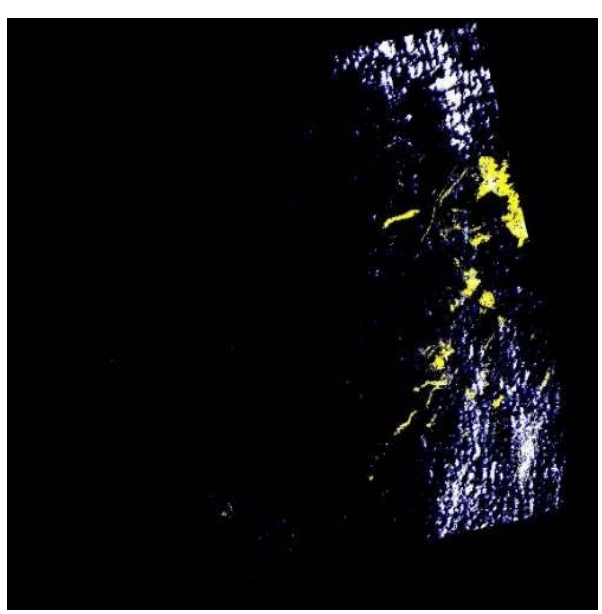

(a)

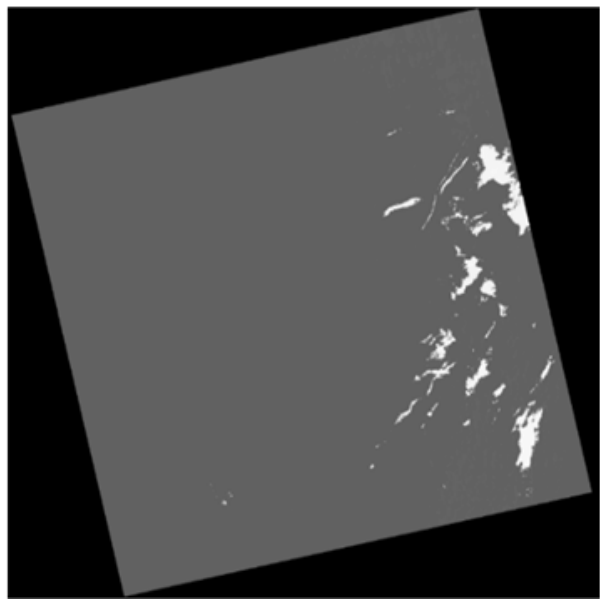

(b)

Fig. 13. QA of Input Image Acquired on 26-04-2017 (a) Quality image with color (b) Quality Assessment Band (QBA) Image. 
These pixel values are integer values which makes difference between different entities like cloud, cirrus, shadow, snow, terrain occlusion etc. Fig. 13 (a) represents Quality image with different colours, as per interpretations of color, one can see here white color represents clouds, yellow is for cirrus, blue is for shadow[https://www.usgs.gov/media/images/landsat-collection-2-full-resolution-browse-quality-imagedesignations] [1].

\begin{tabular}{|l|l|}
\hline \multicolumn{1}{|c|}{ Area } & \multicolumn{1}{c|}{ Pixel Value } \\
\hline Cloud & 7072 \\
\hline Cirrus & 2800 \\
\hline Cloud Shadow & 6816 \\
\hline Cirrus & 2976 \\
\hline Clear Pixels & 2720 \\
\hline
\end{tabular}

Table 3. Cloud and Shadow Pixels

Pixel values of these regions are identified using value plugin in QGIS and by selecting these pixel values from classification image, Cloud and Shadow masks are prepared. Table 3 depicts pixel values identified. Cloud and Shadow mask generated is shown in Fig. 14 (f) for input image acquired on 26-4-2017 by using pixel values identified from QBA.

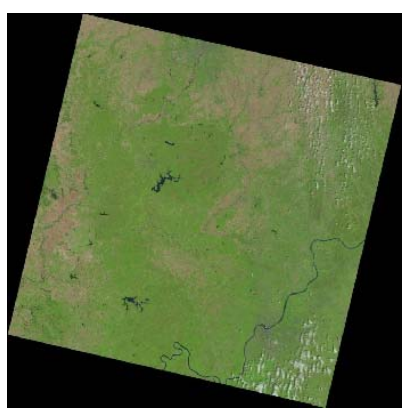

(a) Input Image (26/4/2017)

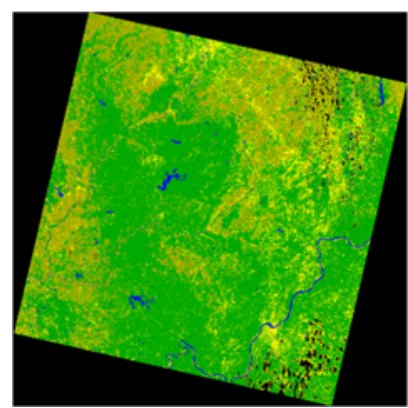

(d) Classification using NDVI of $(26 / 4 / 2017)$

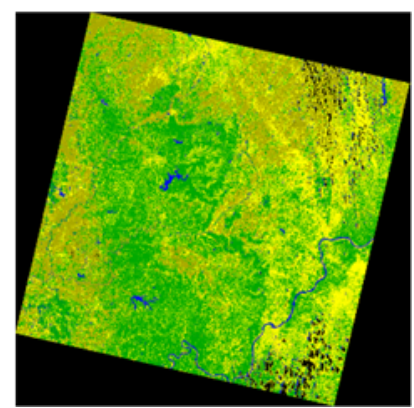

(b) Minimum Distance Classification Image (26/4/2017)

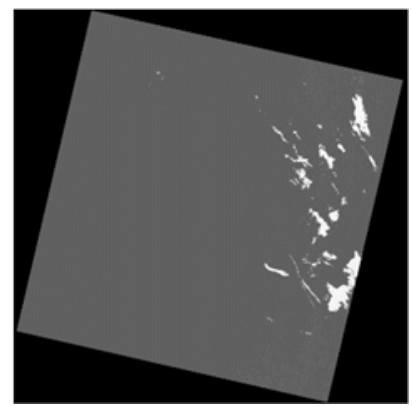

(e) BQA of (26/4/2017)

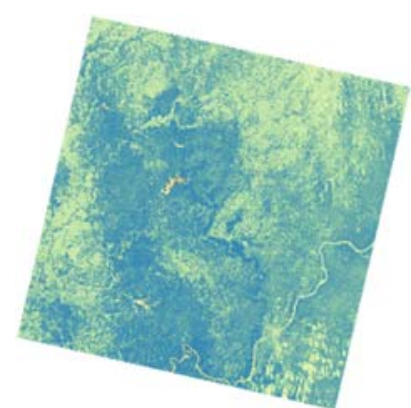

(c) NDVI Calculation Result $(26 / 4 / 2017)$

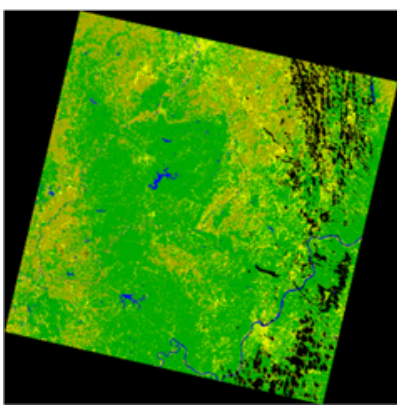

(f) Cloud and Shadow Masking of $(26 / 4 / 2017)$

Fig. 14. Cloud and Shadow Mask Generated for Input Image Acquired on 26-4-2017

By using the method mentioned above proposed system prepares cloud and shadow masks for other input images acquired on 31-7-2017 and 29-4-2018. Fig. 15 shows resultant images required to generate cloud and shadow mask for Input Image Acquired on 31-7-2017. 


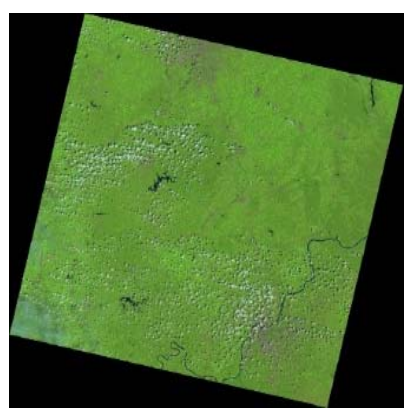

(a) Input Image (31/7/2017)

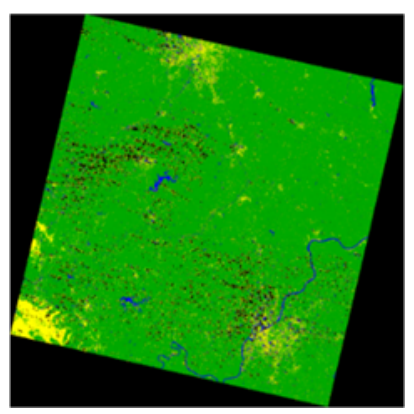

(d) Classification using NDVI of $(31 / 7 / 2017)$

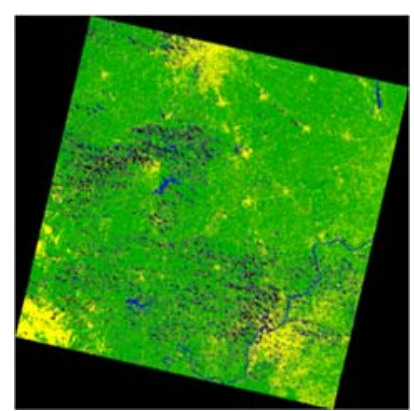

(b) Minimum Distance Classification Image (31/7/2017)

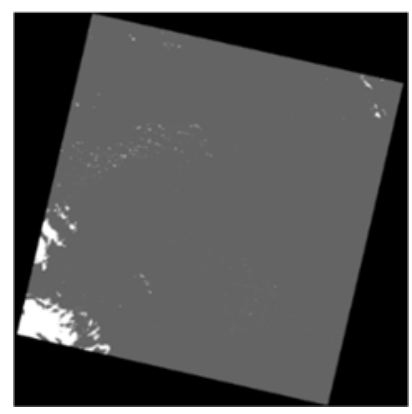

(e) BQA of $(31 / 7 / 2017)$

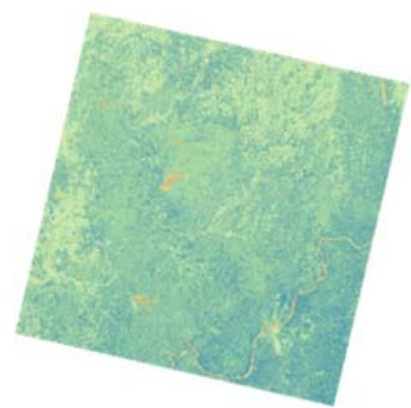

(c) NDVI Calculation Result $(31 / 7 / 2017)$

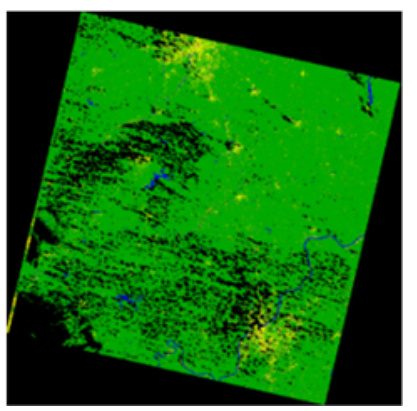

(f) Cloud and Shadow Masking of $(31 / 7 / 2017)$

Fig. 15. Cloud and Shadow Mask Generated for Input Image Acquired on 31-7-2017

Fig. 16 shows resultant images required to generate cloud and shadow mask for Input Image Acquired on 29-42018. This image is called as Reference image used to replace pixels of cloud contamination from 26/4/207 and $31 / 7 / 2017$. This image is having lower cloud cover i.e., $0.3 \%$. Next section shows process of removal and replacement of cloud and shadow contaminated pixels.

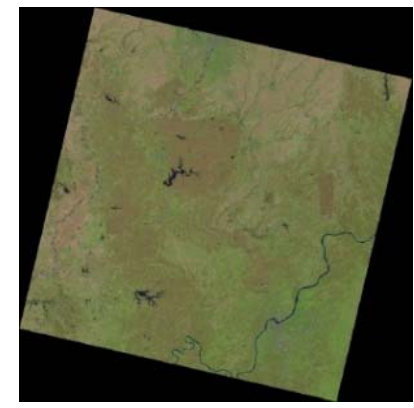

(a) Input Image (29/4/2018)

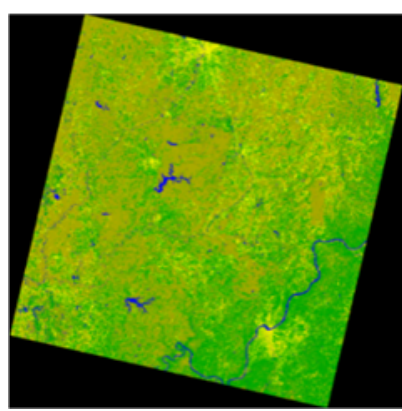

(d) Classification using NDVI of $(29 / 4 / 2018)$

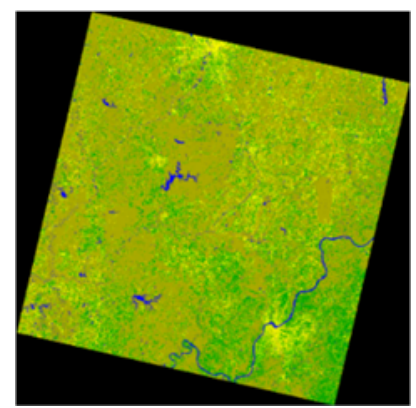

(b) Minimum Distance Classification Image (29/4/2018)

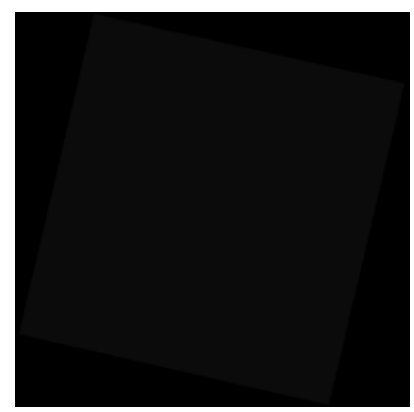

(e) BQA of $(29 / 4 / 2018)$

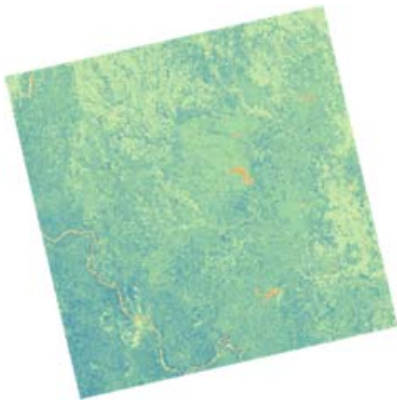

(c) NDVI Calculation Result $(29 / 4 / 2018)$

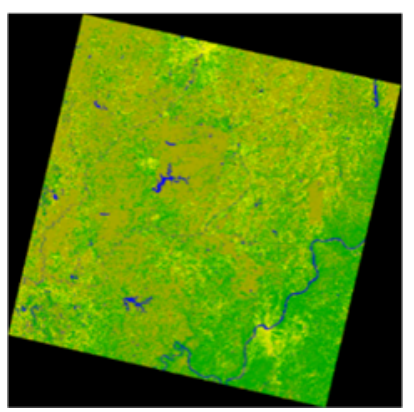

(f) Cloud and Shadow Masking of $(29 / 4 / 2018)$

Fig. 16. Cloud and Shadow Mask Generated for Input Image Acquired on 29-4-2018 


\section{Removal and Replacement of Cloud-Shadow Contaminated Pixels}

From Cloud and Shadow Mask image acquired on 26-4-2017, pixels which are having zero value is identified, then same pixel value from a cloud and Shadow Mask acquired on 29-4-2018

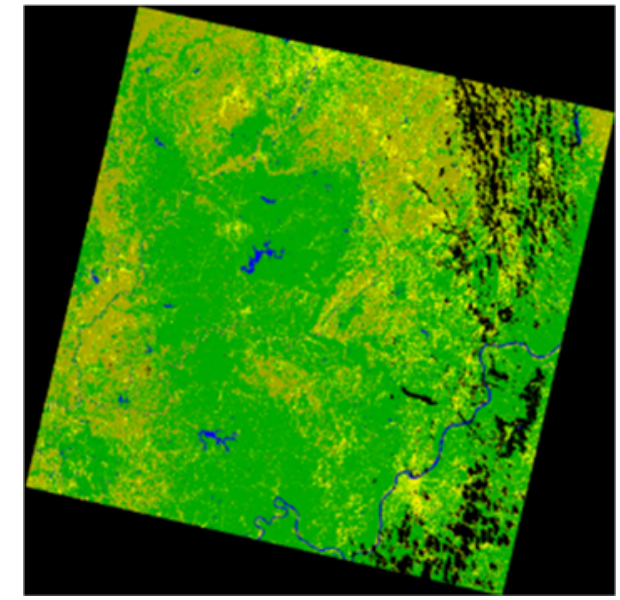

(a)

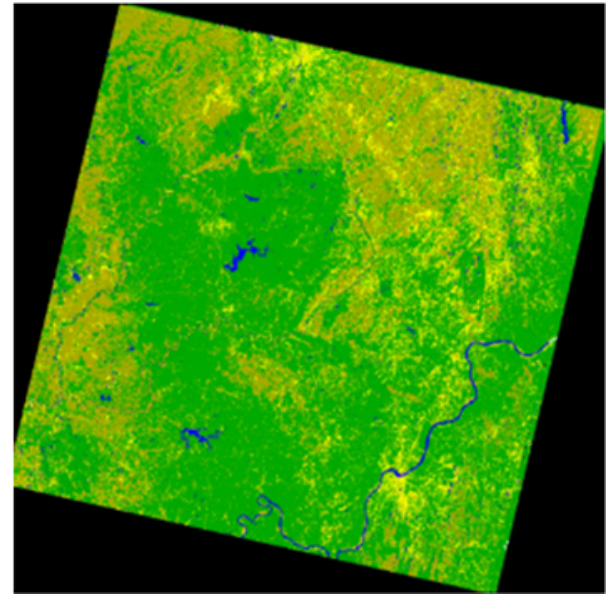

(b)

Fig. 17. Cloud and Shadow Removal from classification image of an Input Image Acquired on 26-4-2017(a) Input Classification Image (b) Cloud and Shadow Removal from Classification image.

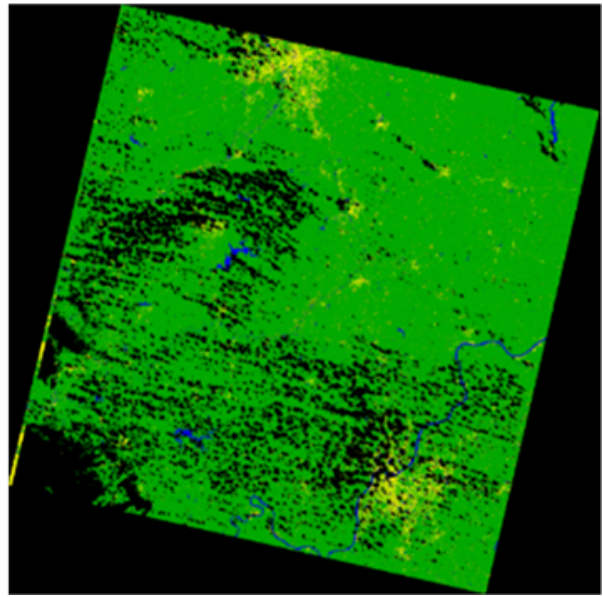

(a)

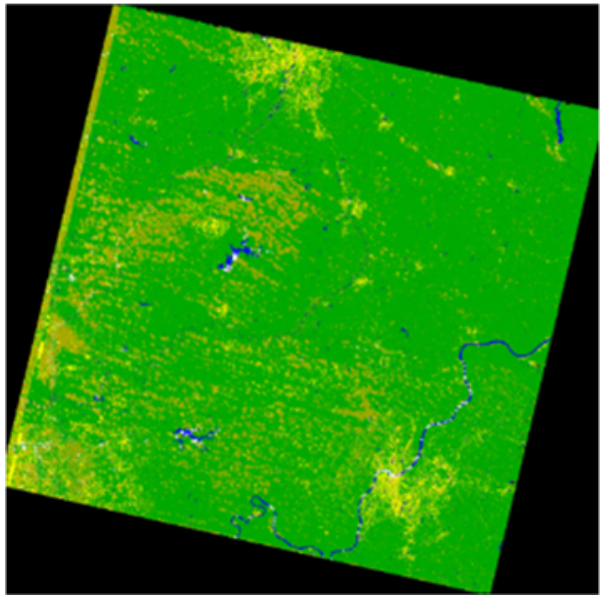

(b)

Fig. 18. Cloud and Shadow Removal from classification image of an Input Image Acquired on 31-7-2017(a) Input Classification Image (b) Cloud and Shadow Removal from Classification image.

Reference image, is checked if it is not zero then pixel of 26-4-2017 is replaced with same pixel 29-4-2018 otherwise if reference image pixel is having zero value, then the pixel of 26-4-2017 is replaced by same pixel of cloud and shadow mask image of 31-7-2017The process shown in Fig. 3 is applied to remove and replace cloud and shadow contaminated pixels.

Same procedure is used to remove pixels with zero values from Cloud and Shadow Mask image of acquired Input Image on 31-7-2017 by using reference image and Cloud and Shadow mask image of acquired Input Image on 26-4-2017

Result of removal of cloud and shadow from classification images are shown in Fig. 17 (b) and Fig. 18 (b). Some of the pixels are remaining unclassified in Fig. 18 (b) as amount of cloud are dense in an Input image acquired on 31-7-2017, so this is one of the limitations of proposed system.

\section{Accuracy Assessment}

An Error Matrix is used for Accuracy assessment calculation pixel wise. Error Matrix represents rows and columns with number of classes present in data. Training data (Map Image) is represented using columns, which is known as true values. 


\begin{tabular}{|c|c|c|c|c|c|c|}
\hline \multirow{2}{*}{\multicolumn{2}{|c|}{ IV_Classified }} & \multicolumn{5}{|c|}{ REFERENCE DATA } \\
\hline & & \multirow{2}{*}{$\begin{array}{l}\text { 1(Built-up) } \\
3746\end{array}$} & \multirow{2}{*}{$\begin{array}{l}\text { 2(Vegetation) } \\
1625 \\
\end{array}$} & \multirow{2}{*}{$\begin{array}{c}\text { 3(Soil) } \\
1974 \\
\end{array}$} & \multirow{2}{*}{$\begin{array}{l}\text { 4(Water) } \\
1327\end{array}$} & \multirow{2}{*}{$\begin{array}{r}\text { Total } \\
8672 \\
\end{array}$} \\
\hline \multirow{5}{*}{ 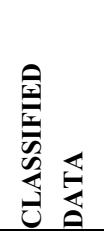 } & 1(Built-up) & & & & & \\
\hline & 2(Vegetation) & 3013 & 128922 & 5831 & 12185 & 149951 \\
\hline & 3(Soil) & 1138 & 290 & 17188 & 536 & 19152 \\
\hline & 4(Water & 10 & 570 & 23 & 25670 & 26273 \\
\hline & Total & 7907 & 131407 & 25016 & 39718 & 204048 \\
\hline \multicolumn{2}{|l|}{ PA } & 47.37 & 98.10 & 68.70 & 64.70 & $69.71 \%$ \\
\hline \multicolumn{2}{|l|}{ UA } & 43.19 & 85.97 & 89.74 & 97.70 & $79.15 \%$ \\
\hline \multicolumn{2}{|c|}{ Errors of Omission Class wise } & $52.7 \%$ & $2 \%$ & $31.2 \%$ & $35.3 \%$ & $30.29 \%$ \\
\hline \multicolumn{2}{|c|}{ Errors of Commission Class wise } & $56.80 \%$ & $14.02 \%$ & $0.10 \%$ & $0.02 \%$ & $17.73 \%$ \\
\hline \multicolumn{2}{|c|}{ Kappa Hat } & \multicolumn{5}{|l|}{0.71} \\
\hline \multicolumn{2}{|c|}{ Accuracy } & \multicolumn{5}{|l|}{$86.02 \%$} \\
\hline
\end{tabular}

Table 4. Accuracy Assessment

Table 5 and Fig. 19 is the comparison of accuracy assessment of different cloud removal techniques used over LANDSAT8/OLI/TIRS data.

\begin{tabular}{|l|l|l|}
\hline & \multicolumn{1}{|c|}{ Cloud Removal Techniques [25][26] } & Overall Accuracy \\
\hline 1 & ACCA (Automated Cloud Cover Assessment) & 83.83 \\
\hline 2 & $\begin{array}{l}\text { ISODATA (Unsupervised Classification with Four Bands, and } \\
\text { clusters used 150) }\end{array}$ & 84.1 \\
\hline 3 & $\begin{array}{l}\text { CFMask T-Cirrus (C implementation of Function of Mask using } \\
\text { Cirrus Band) }\end{array}$ & 85.14 \\
\hline 4 & $\begin{array}{l}\text { SVM (Support Vector Machine) with four bands, samples used in } \\
\text { training - 240 }\end{array}$ & 85.5 \\
\hline 5 & CFMask NT (Nonthermal C implementation of Function of Mask) & 79.15 \\
\hline 6 & $\begin{array}{l}\text { FT-ACCA (Fixed Temperature-Automated Cloud Cover } \\
\text { Algorithm) }\end{array}$ & 74.2 \\
\hline 7 & CART by using four bands and samples in training - 220 & 81.8 \\
\hline 8 & $\begin{array}{l}\text { LAR-CCA (Landsat 8 Surface Reflectance Code (LaSRC) Cloud } \\
\text { Cover Algorithm (CCA)) }\end{array}$ & 73.07 \\
\hline 9 & QUEST four Bands, samples in training - 100 & 84.3 \\
\hline 10 & SEE5 based on Machine Learning and regression & 85.79 \\
\hline 11 & $\begin{array}{l}\text { Proposed Method by using Minimum Distance, NDVI and BQA to } \\
\text { detect and remove Cloud and Shadows with 204,048 training } \\
\text { samples and 7 bands }\end{array}$ & 86.02 \\
\hline
\end{tabular}

Table 5. Analysis on Overall Accuracy of different cloud removal techniques

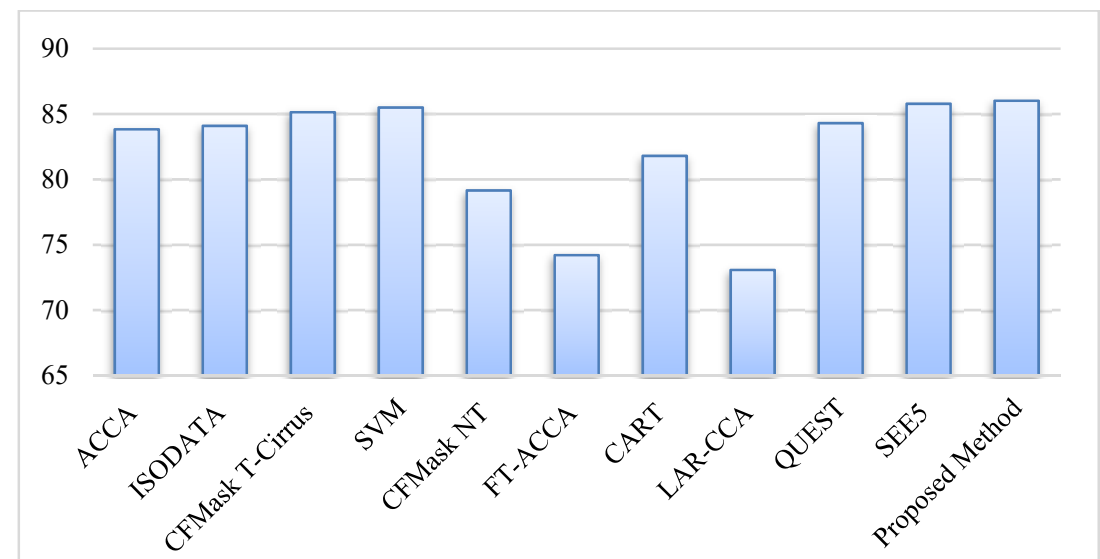

Fig. 19. Comparison of Proposed Method of Cloud and Shadow Removal with Existing Methods. 
The resultant data of mapped classes generated through proposed system is represented in rows. Accuracy assessment [24] is calculated based on values present in an error matrix. Different accuracy metrics are allowed to be calculated by using error matrix. Accuracy metrics such as overall accuracy, UA (User Accuracy), PA (Producer's Accuracy), Kappa Hat Statistics, Omission as well as Commission Errors can be calculated. Reference is Training dataset of Map Image. Classification dataset of pixels (Removed Cloud and Shadow) is assessed with Training dataset. In highlighted rows it shows that these pixels are correctly classified. Accuracy from user perspective is UA (User accuracy) and Accuracy from Producer's Perspective is PA (Producer Accuracy), UA of proposed system is $79.15 \%$ and PA is $69.71 \%$. From User's Perspective accuracy of classification is more than Producer's Perspective. Errors of Omission is $30.29 \%$ which are the reference pixels that were left out (or omitted) from the correct class in the classified map. Error of Commission is $17.73 \%$ which are the pixels that are classified as to reference area that were left out (or omitted) from the correct class in the classified map. Kappa coefficient of 0.71 implies that the classification process was avoiding $71 \%$ of the error that a completely random classification would generate. A value close to 1 indicates that the classification is significantly better than random. Overall Accuracy is $86.02 \%$ which describes that correctly mapped pixels from reference area.

From table 5 and Fig. 19, it can be observed that almost every algorithm can perform well and accuracy achieved for proposed method is also acceptable. In classification algorithms accuracy depends on the number of training samples. Accuracy assessment of proposed method is calculated for 204048 pixels in training data. If number of pixels are increased in training sample, result of accuracy will be more desirable.

\section{Conclusion}

Present article, represents an application of Supervised Classification to Detect and Remove Cloud and Shadow from a Classification Image. Detection and Removal of cloud and shadow is proposed by using combination of Band-2 to Band-7 LANDSAT8 OLI/TIRS. Dark Object Subtraction is used to remove extra darkness occurred due to atmospheric interference. Minimum Distance Algorithm is used for Classification of an Image. Classification is Enhanced with NDVI calculation. BQA is used for pixel identification to prepare Cloud and Shadow Mask. Cloud and Shadow contaminated pixels are removed with Reference Image. Accuracy Assessment is calculated for 204048 pixels in training data. Even though $30.29 \%$ pixels were left in terms of Omission Error and $17.73 \%$ pixels left in commission Error, total Accuracy is more than $85 \%$ for selected Land Cover Scene i.e., $86.02 \%$. Kappa Hat statistics is also closer to one i.e., $0.71 \%$. By comparing proposed system's accuracy with existing algorithms, it can be concluded as, performance of proposed system is acceptable and Overall accuracy can be improved by reducing number of pixels left out in Omission as well as Commission Error.

\section{Acknowledgments}

I sincerely thanks to Luca Congedo, a developer of "Semi Classification Plugin", which really helped to implement a remote sensing application and his contribution in tutorials available on the website "From GIS to Remote Sensing" [2] are very useful for researchers as well as new learners in the field of Remote Sensing.

\section{References}

[1] U.S. Geological Survey home page, www.usgs.gov, last accessed 2021/09/08.

[2] From GIS to Remote Sensing home page, fromgistors.blogspot.com/, last accessed 2021/06/18.

[3] Richards J.A.: Supervised Classification Techniques. In: Remote Sensing Digital Image Analysis. Springer, Berlin, Heidelberg. https://doi.org/10.1007/978-3-642-30062-2_8. (2013)

[4] Pat S. Chavez, An improved dark-object subtraction technique for atmospheric scattering correction of multispectral data, Remote Sensing of Environment, Volume 24, Issue 3, Pages 459-479, ISSN 0034-4257, https://doi.org/10.1016/0034-4257(88)90019-3. (1988)

[5] Image Classification in QGIS home page, https://www.igismap.com/image-classification-in-qgis-supervised-and-unsupervisedclassification/ last accessed 2021/07/31.

[6] D. Jeevalakshmi, S. N. Reddy and B. Manikiam,: Land cover classification based on NDVI using LANDSAT8 time series: A case study Tirupati region, 2016 International Conference on Communication and Signal Processing (ICCSP), 2016, pp. 1332-1335, doi: 10.1109/ICCSP.2016.7754369.

[7] Matlab homepage, https://www.mathworks.com/help/images/ref/ndvi.html, last accessed 2021/05/18.

[8] Image classification home page, http://ecoursesonline.iasri.res.in/mod/page/view.php?id=2065, last accessed 2021/07/31.

[9] N. M. Ngom, M. Mbaye, D. Baratoux, L. Baratoux, T. Catry, N. Dessay, G. Faye, E. H. Sow, E. Delaitre: Mapping Artisanal and SmallScale Gold Mining in Senegal Using Sentinel 2 Data, GeoHealth, https://doi.org/10.1029/2020GH000310, 2020.

[10] Alfieri, J. G., Niyogi, D., Lemone, M. A., Chen, F., and Fall, S., "A Simple Reclassification Method for Correcting Uncertainty in Land Use/Land Cover Data Sets Used with Land Surface Models", <i>Pure and Applied Geophysics</i>, vol. 164, no. 8-9, pp. 1789-1809, 2007. doi:10.1007/s00024-007-0241-4.

[11] Sorour Mohajerani and Parvaneh Saeedi, "Cloud-Net: An end-to-end Cloud Detection Algorithm for Landsat 8 Imagery", journals/corr/abs-1901-10077, 2019, http://arxiv.org/abs/1901.10077, export.arxiv.org

[12] Mateo-García, G., Gomez-Chova, L., Amoros-Lopez, J.,Munoz-Marí, J.,Camps-Valls, G. Multitemporal Cloud Masking in the Google Earth Engine. Remote Sens. 2018, 10, 1079. https://doi.org/10.3390/rs10071079

[13] Jacob Høxbroe Jeppesen, Rune HylsbergJacobsen, Fadil Inceoglu, Thomas SkjødebergToftegaard. "A cloud detection algorithm forsatellite imagery based on deep learning",Remote Sensing of Environment, 2019 
[14] Wang, T., Shi, J., Letu, H., Ma, Y., Li, X.,\& Zheng, Y. (2019). Detection and removal of clouds and associated shadows in satellite imagery based on simulated radiance fields. Journal of Geophysical Research: Atmospheres, 124, 7207-7225. https://doi.org/10.1029/2018JD029960

[15] Hollstein, A.; Segl, K.; Guanter, L.; Brell, M.; Enesco, M. Ready-to-Use Methods for the Detection of Clouds, Cirrus, Snow, Shadow, Water and Clear Sky Pixels in Sentinel-2 MSI Images. Remote Sens. 2016, 8, 666. https://doi.org/10.3390/rs8080666

[16] Gonzalo Mateo-García, Luis Gómez-Chova,Julia Amorós-López, Jordi Muñoz-Marí, GustauCamps-Valls. "Multitemporal Cloud Masking in the Google Earth Engine", Remote Sensing,2018

[17] Andrea Meraner, Patrick Ebel, Xiao Xiang Zhu, Michael Schmitt. "Cloud removal in Sentinel-2 imagery using a deep residual neural network and SAR-optical data fusion", ISPRS Journal of Photogrammetry and Remote Sensing, 2020

[18] Steve Foga, Pat L. Scaramuzza, Song Guo, Zhe Zhu, Ronald D. Dilley, Tim Beckmann, Gail L. Schmidt, John L. Dwyer, M. Joseph Hughes , Brady Laue, "Cloud detection algorithm comparison and validation for operational Landsat data products", Remote Sensing of Environment 194 (2017) 379-390, http://dx.doi.org/10.1016/j.rse.2017.03.026.

[19] Miao Li, Shuying Zang, Bing Zhang, Shanshan Li \& Changshan Wu, "A Review of Remote Sensing Image Classification Techniques: the Role of Spatio-contextual Information", Information, European Journal of Remote Sensing, 47:1, 389-411, DOI: 10.5721 /EuJRS20144723

[20] RI Pyongsop, Ma Z B, Qi Q W and Liu G H., "Cloud and shadow removal from LANDSAT TM data." Journal of Remote Sensing. 14(3): 534-545

[21] D. Cerra, J. Bieniarz, R. M"uller, P. Reinartz,: Cloud Removal from Sentinel-2 Image Time Series Through Sparse Reconstruction From Random Samples, The International Archives of the Photogrammetry, Remote Sensing and Spatial Information Sciences, Volume XLIB3, 2016, XXIII ISPRS Congress, 12-19 July 2016,

[22] Prague, Czech Republiciles M. Foody, Ajay Mathur, toward intelligent training of supervised image classifications: directing training data acquisition for SVM classification, Remote Sensing of Environment, Volume 93, Issues 1-2, 2004, Pages 107-117, ISSN 00344257, https://doi.org/10.1016/j.rse.2004.06.017.

[23] Dongmei Huang, Shoujue Xu, Jingqi Sun, Suling Liang, Wei Song, Zhenhua Wang, : Accuracy assessment model for classification result of remote sensing image based on spatial sampling, J. of Applied Remote Sensing, 11(4), 046023 (2017). https://doi.org/10.1117/1.JRS.11.046023

[24] Fu W., Ma J., Chen P., Chen F. (2020) Remote Sensing Satellites for Digital Earth. In: Guo H., Goodchild M.F., Annoni A. (eds) Manual of Digital Earth. Springer, Singapore. https://doi.org/10.1007/978-981-32-9915-3 3

[25] Li, C.; Wang, J.; Wang, L.; Hu, L.; Gong, P. Comparison of Classification Algorithms and Training Sample Sizes in Urban Land Classification with Landsat Thematic Mapper Imagery. Remote Sens. 2014, 6, 964-983. https://doi.org/10.3390/rs6020964

[26] Steve Foga, Pat L. Scaramuzza, Song Guo, Zhe Zhu, Ronald D. Dilley, Tim Beckmann, Gail L. Schmidt, John L. Dwyer, M. Joseph Hughes , Brady Laue, "Cloud detection algorithm comparison and validation for operational Landsat data products", Remote Sensing of Environment 194 (2017) 379-390, http://dx.doi.org/10.1016/j.rse.2017.03.026.

[27] G. Xudong, C. Huang, L. Gaohuan and L. Qingsheng, "Integrating time-series and high-spatial remote sensing data based on multilevel decision fusion," IGARSS 2020 - 2020 IEEE International Geoscience and Remote Sensing Symposium, 2020, pp. 212-215, doi: 10.1109/IGARSS39084.2020.9323564.

[28] Landsat Mission home page landsat.usgs.gov last accessed 2021/09/08

[29] Congedo, L., (2021). Semi-Automatic Classification Plugin: A Python tool for the download and processing of remote sensing images in QGIS. Journal of Open Source Software, 6(64), 3172. https://doi.org/10.21105/joss.03172

\section{Authors Profile}

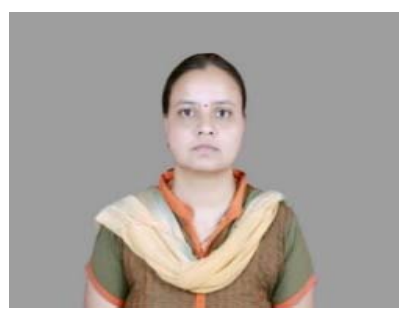

Renuka S. Gound, is a Research Scholar at Pimpri Chinchwad College of Engineering, Pune and has more than 13 years of experience of teaching in Information Technology Department of Pimpri Chinchwad College of Engineering. Completed BE in Computer Science and Engineering in 2005 and M. Tech in Computer Engineering in 2012. Published more than 25 papers in journals and conferences. Research Area of Interest is Processing Satellite Images, Machine Learning, Remote Sensing, Computer Vision.

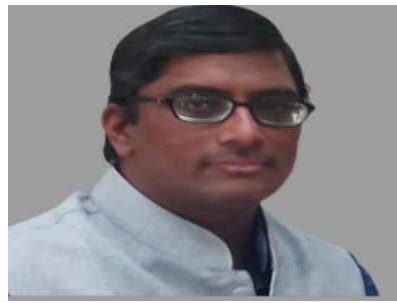

Dr. Sudeep D. Thepade, Dean of Quality Assurance and Professor in Computer Engineering Department, at Pimpri Chinchwad College of Engineering, Pune. He has more than 330 research publications in renowned Journals and Conferences. He is member of BoS of Information Technology department at SPPU. He has 19+ years of experience in the field of education. His Area of Interest is Image processing, Computer Vision, Deep Learning. He has many Best Paper Awards to his credit. Two scholars have completed Ph.D. under his supervision and six are pursuing. He has guided more than $25 \mathrm{M}$. Tech/ ME projects and several BE projects. 